\title{
Transcriptional regulation by infliximab therapy in kawasaki disease patients with immunoglobulin resistance
}

\author{
Yoshihito Ogihara', Shohei Ogata' ${ }^{1}$ Keiko Nomoto' ${ }^{1}$, Takasuke Ebato ${ }^{1}$, Kayoko Sato $^{1}$, Kenichi Kokubo ${ }^{2}$, Hirosuke Kobayashi ${ }^{2}$ and \\ Masahiro Ishii'
}

BACKGROUND: Infliximab (IFX), a known monoclonal antibody against tumor necrosis factor-a (TNF-a), is used to treat Kawasaki disease (KD) patients with intravenous immunoglobulin (IVIG) resistance. The transcriptional modulation of inflammation following IFX therapy has not been reported in KD patients.

METHODS: We investigated the transcript abundance profiles in whole blood obtained from eight IVIG-resistant KD subjects treated with IFX therapy using microarray platforms and compared them with those in initially IVIG-responsive subjects. A pathway analysis was performed using WikiPathways to search for the biological pathways of the transcript profiles. Four transcripts changed by IFX therapy were subsequently validated using quantitative real-time polymerase chain reaction.

RESULTS: The pathway analysis showed the reduced abundance of transcripts in the nucleotide-binding oligomerization domain, matrix metalloproteinase (MMP), and inflammatory cytokine pathways and the increased abundance of transcripts in the T-cell receptor, apoptosis, TGF- $\beta$, and interleukin-2 pathways. Additionally, the levels of four transcripts (peptidase inhibitor-3, MMP-8, chemokine receptor-2, and pentraxin-3) related to KD vasculitis and IVIG resistance decreased after IFX therapy.

CONCLUSION: The administration of IFX was associated with both the signaling pathways of KD inflammation and several transcripts related to IVIG resistance factors. These findings provide strong theoretical support for the use of IFX in KD patients with IVIG resistance.

$\mathbf{T}$ he severest complication of Kawasaki disease (KD) is the development of coronary artery aneurysms (CAAs). Although the administration of high-dose intravenous immunoglobulin (IVIG) and oral aspirin can be used to reduce systemic inflammation and the duration of fever, approximately $10 \%$ to $20 \%$ of KD patients exhibit a persistent fever despite receiving initial IVIG treatment (1). IVIG resistance is a risk factor for CAA. Therefore, the optimal treatment for IVIGresistant $\mathrm{KD}$ must be selected.

Tumor necrosis factor- $\alpha$ (TNF- $\alpha$ ) is an important cytokine that induces an inflammatory status in KD patients. In previous reports, the serum levels of TNF- $\alpha$ and soluble TNF receptor 1 have been found to be significantly elevated in KD patients during the acute phase, whereas the levels of circulatory TNF- $\alpha$ are significantly higher in KD patients with CAAs than in those without CAAs $(2,3)$. In addition, it has been demonstrated that TNF- $\alpha$ is involved in thedevelopment of CAAs in an animal model of KD (4). Therefore, the blocking of TNF- $\alpha$ is a critical factor for regulating the KD inflammation.

Infliximab (IFX), a monoclonal antibody that blocks the biological activity of TNF- $\alpha$ when administered intravenously, is used to treat several chronic inflammatory diseases, including Crohn's disease and rheumatoid arthritis (5). Recently, the use of IFX has been attempted as a novel therapy for refractory KD (6-9). In a randomized study of additional IVIG vs. IFX infusion in KD patients with fever following initial treatment with IVIG, IFX infusion was found to be safe and well tolerated in KD patients, with no serious adverse reactions attributed to either therapy. Another retrospective study demonstrated that IFX therapy, as a second-line treatment, resulted in faster disease resolution with a reduced number of days of hospitalization. Furthermore, the outcomes of patients with CAAs are similar compared with those of patients treated with additional IVIG therapy $(7,8)$. Although it is known that the blocking of TNF- $\alpha$ is critical for obtaining anti-inflammatory effects in patients with $\mathrm{KD}$, the transcriptional modulation of inflammation following the administration of IFX therapy has not been previously reported in the setting $\mathrm{KD}$, and regarding the different mechanisms between IFX and IVIG therapies for KD remains unknown. Therefore, to investigate the mechanism of action of IFX therapy in the setting of KD, we detected the transcript profiles in KD patients treated with infused IFX using a microarray platform and quantitative real-time polymerase chain reaction (RT-PCR).

\section{RESULTS}

Transcript Abundance Profiles and Signaling Pathways Associated With IFX Therapy in IVIG-Resistant Subjects

To demonstrate the differential transcript abundance profiles observed in patients treated with IFX therapy, we first identified the transcript abundance profiles in IFX-responsive $\mathrm{KD}$ 
subjects $(n=8)$ who received IFX therapy as the third-line treatment after two cycles of IVIG therapy (Figure 1 and Table 1 ). The transcript expression levels of 7,244 probes changed significantly between the pre- and post-IFX therapy periods $(P<$ 0.05). To focus on the most significant differentially expressed

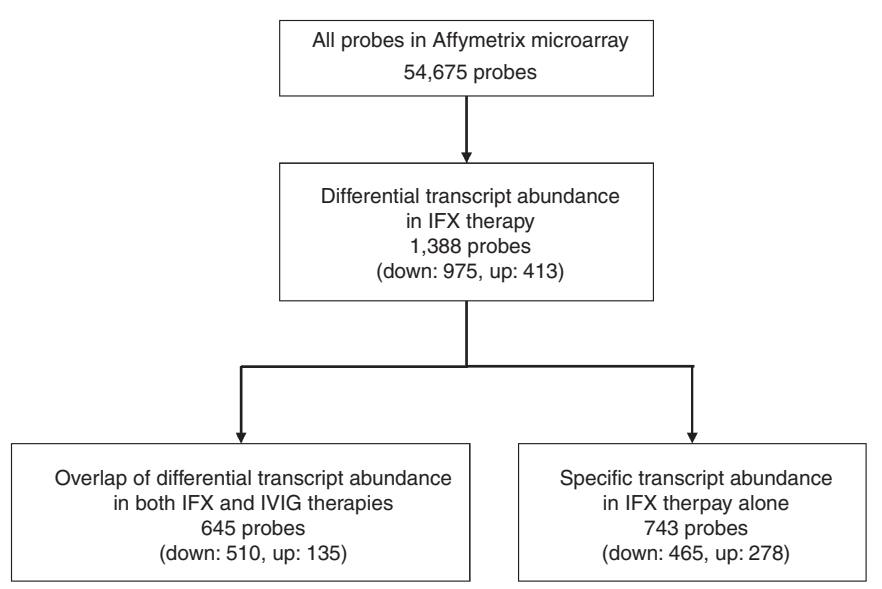

Figure 1. Flow diagram of the transcripts in the patients treated with IFX therapy. Among 1,388 probes that changed significantly, 975 were less abundant (downregulated) and 413 were more abundant (upregulated) after IFX therapy ( $p<0.05,>1.5$-fold change). After excluding 645 overlapping probes that were differentially abundant in both IFX and IVIG therapy groups (downregulated: 510, upregulated: 135), a total of 743 probes that specifically changed under IFX therapy alone were identified (downregulated: 465 , upregulated: 278 ). All eight subjects responded to IFX therapy after a total dose of $4 \mathrm{~g} / \mathrm{kg}$ of IVIG. All six subjects responded to the initial IVIG therapy. IFX, infliximab; IVIG, intravenous immunoglobulin. transcripts among the various phases of IFX therapy, we selected 1,388 of 7,244 probes whose expression levels in the pre-IFX therapy subjects exhibited a more than 1.5 -fold change in the post-IFX therapy period. Among these 1,388 probes, 975 (70.2\%) were less abundant (downregulated) and 413 (29.8\%) were more abundant (upregulated), with a more than 1.5 -fold change, after IFX therapy. Table 2 shows the top 20 probes (downregulated and upregulated probes, respectively) with the largest fold differences in their transcript abundance profiles between the pre- and post-IFX therapy periods (maximum $Q$ value: $4.9 \%$ ). The most downregulated transcripts among the subjects who received IFX therapy were CD177 (polycythemia rubra vera-1), peptidase inhibitor-3 (PI3), suppressor of cytokine signaling 3, interleukin-1 (IL-1) receptor type 2 (IL1R2), matrix metalloproteinase 8 (MMP8), and IL-1 $\beta$. Among the most significantly upregulated transcripts in the subjects who received IFX therapy, those associated with hemoglobin synthesis, intercellular matrix regulation and nucleic acid metabolism were included. Moreover, some TGF- $\beta$-related transcripts, including TGF- $\beta$ receptor III and noggin were more abundant after IFX therapy.

Table 3 shows the signaling pathways associated with the downregulated and upregulated probes. Among the 975 downregulated probes in the subjects who received IFX therapy, a total of 80 signaling pathways were detected in association with a broad range of genes, such as those involving nucleotide-binding oligomerization domain (NOD, $P=4.12 \times 10^{-10}$ ), type II interferon (IFN- $\left.\gamma, P=1.81 \times 10^{-8}\right)$, IL-1 $\left(P=9.99 \times 10^{-8}\right)$, Toll-like receptor (TLR, $P=4.08 \times 10^{-7}$ ), complement and

Table 1. Clinical characteristics and laboratory values of the subjects pre- and post-IFX therapy

\begin{tabular}{|c|c|c|c|c|c|c|}
\hline Clinical characteristics & \multicolumn{3}{|c|}{ Subjects for microarray analysis $(n=8)$} & \multicolumn{3}{|c|}{ Subjects for RT-PCR $(n=9)$} \\
\hline Male, $n(\%)$ & \multicolumn{3}{|c|}{$6(75)$} & \multicolumn{3}{|c|}{$5(56)$} \\
\hline Age at onset, years & \multicolumn{3}{|c|}{$4.2(1.5-7.0)^{\mathrm{a}}$} & \multicolumn{3}{|c|}{$4.5(1.6-5.9)$} \\
\hline Illness days of IFX, days & \multicolumn{3}{|c|}{$10.5(8-12)$} & \multicolumn{3}{|c|}{$11.0(8-12)$} \\
\hline Laboratory values & Pre-IFX & Post-IFX & $P$ & Pre-IFX & Post-IFX & $P$ \\
\hline $\mathrm{WBC}, \times 10^{3} / \mathrm{mm}^{3}$ & $16.8(13.7-21.0)^{b}$ & $11.0(9.5-13.0)$ & 0.01 & $13.8(10.9-18.7)$ & $10.7(7.6-12.4)$ & 0.01 \\
\hline Neutrophil, $\times 10^{3} / \mathrm{mm}^{3}$ & $11.0(9.7-15.3)$ & $5.7(4.6-8.3)$ & 0.01 & $9.7(7.6-12.7)$ & $5.4(3.1-6.5)$ & 0.01 \\
\hline Lymphocyte, $\times 10^{3} / \mathrm{mm}^{3}$ & $4.1(3.1-5.0)$ & $4.8(3.4-5.0)$ & NS & $3.9(2.8-4.2)$ & $4.4(3.6-5.0)$ & NS \\
\hline AST, IU/I & $28.0(21.5-33.5)$ & $31.5(25.3-46.5)$ & 0.04 & $27.0(22.0-29.0)$ & $27.0(25.5-37.5)$ & 0.03 \\
\hline ALT, IU/I & $16.5(10.3-42.3)$ & $16.5(10.8-55.5)$ & NS & $13.0(10.5-25.5)$ & $14.0(12.0-17.5)$ & NS \\
\hline $\mathrm{CRP}, \mathrm{mg} / \mathrm{dl}$ & $8.7(5.1-11.6)$ & $3.6(2.5-4.6)$ & 0.01 & $8.7(3.5-12.5)$ & $4.0(2.4-7.0)$ & 0.01 \\
\hline
\end{tabular}

$P$ values were calculated by the Wilcoxon signed-rank test for continuous variables.

AST, aspartate aminotransferase; ALT, alanine aminotransferase; CAAs, coronary artery aneurysms ( $\geq 5 Z$-max); CRP, C-reactive protein; IFX, infliximab; IIIness day 1, first calendar day of

fever; IQR, interquartile range; NS, not significant; WBC, white blood cells; Z-max, maximum Z-score in any coronary artery segment during 1 month after onset.

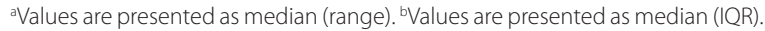


Table 2. Top 20 probes with the largest fold differences in transcript abundance profiles between pre- and post-IFX therapy

\begin{tabular}{|c|c|c|c|c|c|c|c|}
\hline Probe ID & Gene symbol & Gene name & $\begin{array}{l}\text { Fold change } \\
\text { (pre-/post-IFX) }\end{array}$ & $P$ & $Q(\mathrm{FDR})$ & IFX & IFX and IVIG \\
\hline \multicolumn{8}{|c|}{ Downregulated probes } \\
\hline 41469_at & $P I 3$ & Peptidase inhibitor 3, skin-derived & 3.44 & 0.012 & 0.019 & + & \\
\hline 219607_s_at & MS4A4A & $\begin{array}{l}\text { Membrane-spanning 4-domains, subfamily } A \text {, } \\
\text { member } 4 A\end{array}$ & 3.42 & 0.012 & 0.019 & + & \\
\hline 202437_s_at & CYP1B1 & Cytochrome P450, family 1, subfamily B, polypeptide 1 & 3.31 & 0.012 & 0.019 & & + \\
\hline 205844_at & VNN1 & Vanin 1 & 3.23 & 0.012 & 0.019 & & + \\
\hline 225207_at & PDK4 & Pyruvate dehydrogenase kinase, isozyme 4 & 3.18 & 0.025 & 0.028 & + & \\
\hline 203691_at & $P 13$ & Peptidase inhibitor 3, skin-derived & 3.14 & 0.012 & 0.019 & + & \\
\hline 215783_s_at & $A L P L$ & Alkaline phosphatase, liver/bone/kidney & 2.91 & 0.012 & 0.019 & + & \\
\hline 202436_s_at & CYP1B1 & Cytochrome P450, family 1 , subfamily B, polypeptide 1 & 2.89 & 0.012 & 0.019 & & + \\
\hline 202435_s_at & CYP1B1 & Cytochrome P450, family 1 , subfamily B, polypeptide 1 & 2.83 & 0.012 & 0.019 & & + \\
\hline 211372_s_at & IL1R2 & Interleukin 1 receptor, type II & 2.82 & 0.012 & 0.019 & & + \\
\hline 1555728_a_at & MS4A4A & $\begin{array}{l}\text { Membrane-spanning 4-domains, subfamily } A \text {, } \\
\text { member } 4 A\end{array}$ & 2.78 & 0.012 & 0.019 & + & \\
\hline 207329_at & MMP8 & Matrix metalloproteinase 8 (neutrophil collagenase) & 2.62 & 0.012 & 0.019 & + & \\
\hline 205067_at & $I L 1 B$ & Interleukin $1 \beta$ & 2.62 & 0.012 & 0.019 & & + \\
\hline 1552554_a_at & NLRC4 & NLR family, CARD domain containing 4 & 2.60 & 0.012 & 0.019 & + & \\
\hline 236081_at & SNCA & Synuclein $\alpha$ (non A4 component of amyloid precursor) & 0.42 & 0.012 & 0.032 & & + \\
\hline 223124_s_at & C1orf128 & PITH domain containing 1 & 0.42 & 0.012 & 0.032 & & + \\
\hline 204720_s_at & DNAJC6 & DnaJ (Hsp40) homolog, subfamily C, member 6 & 0.44 & 0.012 & 0.032 & & + \\
\hline 241881_at & OR2W3 & Olfactory receptor, family 2 , subfamily W, member 3 & 0.45 & 0.036 & 0.041 & & + \\
\hline 204731_at & TGFBR3 & Transforming growth factor, $\beta$ receptor III & 0.46 & 0.012 & 0.032 & + & \\
\hline 223670_s_at & HEMGN & Hemogen & 0.46 & 0.017 & 0.032 & & + \\
\hline 230720_at & RNF182 & Ring finger protein 182 & 0.46 & 0.012 & 0.032 & & + \\
\hline 203502_at & $B P G M$ & 2,3-Bisphosphoglycerate mutase & 0.46 & 0.036 & 0.041 & & + \\
\hline 231798_at & NOG & Noggin & 0.47 & 0.012 & 0.032 & + & \\
\hline 220646_s_at & KLRF1 & Killer cell lectin-like receptor subfamily F, member 1 & 0.47 & 0.049 & 0.049 & + & \\
\hline 220173_at & C14orf45 & Coiled-coil domain containing 176 & 0.48 & 0.012 & 0.032 & & + \\
\hline 37117_at & ARHGAP8 & Rho GTPase activating protein 8 & 0.48 & 0.025 & 0.037 & & + \\
\hline 214470_at & KLRB1 & Killer cell lectin-like receptor subfamily $B$, member 1 & 0.48 & 0.025 & 0.037 & + & \\
\hline 243508_at & C20orf196 & Chromosome 20 open reading frame 196 & 0.48 & 0.012 & 0.037 & & + \\
\hline 221747_at & TNS1 & Tensin 1 & 0.49 & 0.049 & 0.049 & & + \\
\hline 235276_at & EPSTI1 & Epithelial stromal interaction 1 & 0.49 & 0.012 & 0.032 & + & \\
\hline
\end{tabular}

P values were calculated by the Mann-Whitney U-test. Q values were calculated by the Benjyamini Hochberg formula. IFX and IVIG:The probes were less or more abundant after IFX therapy in the eight subjects who responded to the IFX therapy and after IVIG therapy in the six subjects who responded to the initial IVIG therapy.

FDR, false discovery rate; IFX, infliximab; IVIG, intravenous immunoglobulin. 


\section{Articles | ogihara et al.}

coagulation cascades $\left(P=3.40 \times 10^{-6}\right)$, IL-6 $\left(P=1.12 \times 10^{-4}\right)$, TNF- $\alpha(P=0.001)$, and MMPs $(P=0.002)$, which encode proteins expressed at the cell surface, in the cytoplasm, and in the nucleus; these pathways were markedly suppressed by IFX therapy. Among the 413 upregulated probes in the subjects who received IFX therapy, a total of 57 signaling pathways were detected, related to the activation of T-cell receptor (TCR, $P<$ $\left.1.00 \times 10^{-10}\right)$, apoptosis $\left(P=2.59 \times 10^{-7}\right)$, TGF- $\beta\left(P=5.90 \times 10^{-5}\right)$, and IL-2 $\left(P=2.99 \times 10^{-4}\right)$.

\section{Expression of Transcript Abundance Profiles Associated With IFX Therapy Compared With IVIG Therapy}

Finally, to identify the specific transcript profiles that are differentially abundant in patients treated with IFX therapy, we excluded transcripts that changed significantly in subjects who were initially IVIG-responsive subjects $(n=6)$, from among the 1,388 probes that changed in the IFX-responsive subjects $(n=8)$ (Figure 1). A total of 645 probes (46.5\%) significantly changed under the IVIG therapy ( $P<0.05,>1.5$-fold change). A total of 743 probes (53.5\%) that were differentially abundant under IFX therapy only were identified after excluding 645 probes that were differentially abundant under IVIG therapy.

Among the 975 probes that were downregulated by IFX therapy, $465(47.7 \%)$ were specifically downregulated in the IFX-responsive subjects and 510 (52.3\%) were downregulated in both the patients treated with IFX and IVIG therapy. In the pathway analysis, a total of 72 and 68 signaling pathways were significantly detected in association with the 465 probes downregulated under IFX therapy and 510 probes downregulated under both therapies, respectively. Most of the signaling pathways suppressed under IFX therapy primarily involved NOD, interferon- $\gamma$, and MMPs, and signaling pathways associated with IL-1, TLR, complement and coagulation cascades, IL- 6 and TNF- $\alpha$ were significantly downregulated under both therapies (Table 2).

A total of 413 probes were upregulated by IFX therapy: 278 probes $(67.3 \%)$ were specifically upregulated under IFX therapy and 135 probes (32.7\%) were upregulated under both IFX and IVIG therapies (Figure 1). In the pathway analysis, a total of 56 and 16 signaling pathways were significantly detected in association with 278 probes in the patients treated with IFX therapy and 135 probes in patients treated with both therapies, respectively. Most of the signaling pathways were upregulated under IFX therapy involved TCR, apoptosis, TGF- $\beta$, and IL-2 (Table 2). No characteristic pathways were upregulated under both therapies.

To identify the specific transcripts that were differentially abundant between the pre- and post-IFX therapy, we selected 50 probes that were more abundant after IVIG therapy from among the 465 probes that were significantly less abundant after IFX therapy alone. These 50 probes included PI3 (3.44fold change), MMP8 (2.62-fold change), chemokine (C-C motif) receptor-2 (CCR2, 1.71-fold change), and pentraxin-3 (PTX3, 1.62-fold change), which play a role in KD vasculitis and IVIG resistance (Supplementary Table S1 online). Among the 278 probes that were more significantly abundant
Table 3. Signaling pathways related to transcripts regulated by IFX therapy

\begin{tabular}{|c|c|c|c|}
\hline Signaling pathway & $P$ & IFX & IFX and IVIG \\
\hline \multicolumn{4}{|l|}{ Downregulated pathways } \\
\hline NOD & $4.12 \mathrm{E}-10$ & + & \\
\hline Type II interferon signaling & $1.81 \mathrm{E}-8$ & + & \\
\hline IL-1 signaling & $9.99 \mathrm{E}-8$ & & + \\
\hline TLR signaling & $4.08 \mathrm{E}-7$ & & + \\
\hline $\begin{array}{l}\text { Complement and } \\
\text { coagulation cascades }\end{array}$ & $3.40 \mathrm{E}-6$ & & + \\
\hline IL-6 signaling & $1.12 \mathrm{E}-4$ & & + \\
\hline TNF- $\alpha$ signaling & 0.001 & & + \\
\hline IL-17 signaling & 0.002 & & + \\
\hline Matrix metalloproteinases & 0.002 & + & \\
\hline \multicolumn{4}{|l|}{ Upregulated pathways } \\
\hline TCR signaling & $<1.00 \mathrm{E}-10$ & + & \\
\hline Apoptosis & $2.59 \mathrm{E}-7$ & + & \\
\hline TGF- $\beta$ signaling & $5.90 \mathrm{E}-5$ & + & \\
\hline IL-2 signaling & $2.99 \mathrm{E}-4$ & + & \\
\hline $\begin{array}{l}\text { Interferon-induced } \\
\text { apoptosis }\end{array}$ & $3.32 \mathrm{E}-4$ & + & \\
\hline \multicolumn{4}{|c|}{$\begin{array}{l}P \text { values in the pathway analysis were calculated by Fisher's exact test. IFX and IVIG: } \\
\text { The signaling pathways for the transcripts that were less or more abundant after IFX } \\
\text { therapy in the eight subjects who responded to IFX therapy and after IVIG therapy in } \\
\text { the six subjects who responded to the initial IVIG therapy. } \\
\text { IFX, infliximab; IVIG, intravenous immunoglobulin. }\end{array}$} \\
\hline
\end{tabular}

after IFX therapy alone, no characteristic transcripts were less abundant after IVIG therapy.

\section{Validation of the Expression of the Transcripts Regulated Under IFX Therapy Using Quantitative RT-PCR}

We selected four transcripts (PI3, MMP8, CCR2, and PTX3) associated with KD inflammation, IVIG resistance and factors induced by TNF- $\alpha$ stimulation from among the 50 probes that were significantly less abundant after IFX therapy but more abundant after IVIG therapy and subsequently validated the expression levels of these transcripts using quantitative RT-PCR with whole blood samples obtained from an independent cohort of IFX-responsive subjects $(n=9)$. The expression levels of PI3, MMP8, CCR2, and PTX3 significantly decreased after IFX therapy (PI3, $P=0.008$; MMP8, $P=0.012$; CCR2, $P=$ 0.008; PTX3, $P=0.038$; Figure 2).

\section{DISCUSSION}

To investigate the mechanism of action of IFX therapy in KD patients, the transcript abundance profiles in whole blood obtained from IFX-responsive KD subjects who received IFX as the third-line treatment after two cycles of IVIG therapy were analyzed before and after IFX therapy. In this study, the transcript abundance, which was found to be related to signaling pathways of $\mathrm{KD}$ inflammation, such as those involving IL-1, IL-6, and TNF- $\alpha$, changed significantly following the administration of IFX therapy. Remarkably, the expression 
a

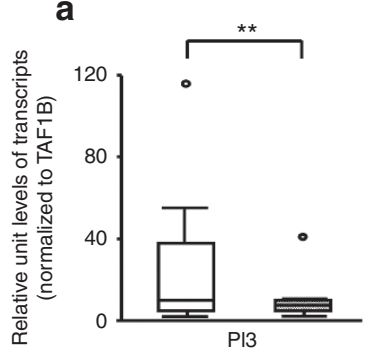

b

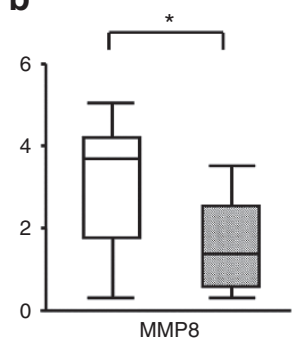

C

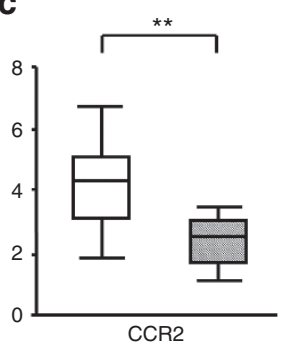

d

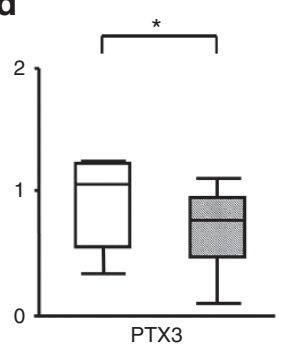

Figure 2. Expression levels of the transcripts among the nine subjects who responded to IFX therapy after two cycles of IVIG therapy. The whole blood transcript expression levels of (a) PI3, (b) MMP8, (c) CCR2, and (d) PTX3 before the IFX (white bars) and 36 h after the end of IFX (gray bars) therapy. The results are presented as relative units of each transcript compared with that of TAF1B. The box plots demonstrate the median and 25th and 75th percentiles, with the whiskers indicating the maximum and minimum values. $P$ values were calculated using the Mann-Whitney $U$-test. ${ }^{*} P<0.05$, ${ }^{* *} P<$ 0.01. CCR2, chemokine receptor-2; peptidase inhibitor-3; MMP8, matrix metalloproteinase-8; PI3, peptidase inhibitor-3; PTX3, pentraxin-3; TAF1B, TATA box-binding protein-associated factor RNA polymerase 1B.

levels of some transcripts associated with IVIG resistance were changed by IFX therapy. These results suggest that IFX therapy regulates important cytokine signal activities involved in $\mathrm{KD}$ inflammation by the blocking of TNF- $\alpha$, which may be critical for regulating IVIG resistance factors.

The administration of IFX was found to be associated with several pathways of innate immunity, such as NOD and TLR; inflammatory cytokines, such as interferon- $\gamma$, IL- 1 , IL-6, TNF$\alpha$, and IL-17; MMPs pathways (downregulated pathways); and TGF- $\beta$ pathways (upregulated pathways) (Table 3 ).

Inflammatory cytokines, such as interferon- $\gamma$, IL-1, IL-6, TNF- $\alpha$, and IL-17, are considered main inflammatory cytokines in $\mathrm{KD}$ and play a role in the onset of $\mathrm{KD}$ vasculitis, CAAs, and IVIG resistance (2,10-12). The increased transcript abundance of several genes in the IL-1 pathway has been confirmed among IVIG-resistant KD subjects (13). Meanwhile, another study reported that the serum levels of soluble TNF receptor 1 and IL- 6 are dramatically decreased after IFX therapy in refractory KD subjects (9). In this study, IFX therapy greatly suppressed the expression levels of transcripts, which are involved in the main pathways of cytokine signaling related to KD vasculitis. Moreover, these inflammatory cytokinerelated pathways were significantly altered by IVIG therapy in the initially IVIG-responsive subjects. These results suggest that the administration of IFX is associated with transcript abundance regarding the pathway of KD inflammation, which can be sufficiently controlled with IVIG therapy, but not with IVIG therapy.

In contrast, NOD, MMPs, and TGF- $\beta$ pathways were changed more significantly by IFX therapy than by IVIG therapy (Table 3). In recent studies, the involvement of TLR signaling and NOD-like receptor signaling in the pathophysiology of the acute phase of $\mathrm{KD}$ has been considered $(14,15)$. In addition, based on a study reporting the repression of increases in the expression of NOD1 and NOD2 mRNA in mice macrophages by anti-TLR or anti-TNF- $\alpha$ antibodies, it can be assumed that the expression of NOD mRNA results from TNF- $\alpha$ production, possibly via TLR signaling (16). The present findings suggest that specific TNF- $\alpha$ blocking with IFX therapy plays an important role in the activation of innate immunity, a crucial factor related to the acute phase of KD. MMPs are family members of zinc-dependent proteinases, proteins secreted from various cells, and break down the protein components of the extracellular matrix (17). Polymorphisms of some MMP genes are associated with disease susceptibility, severity of CAAs in patients with $\mathrm{KD}$ $(18,19)$. Other studies have reported the importance of the TGF- $\beta$ signaling pathway in the pathology of KD vasculitis, based on recent findings that TGF- $\beta$ gene polymorphisms are relevant to the susceptibility to $\mathrm{KD}$ and CAA formation $(20,21)$. These findings suggest that the administration of IFX is associated with several pathways responsible for $\mathrm{KD}$ inflammation and CAA formation.

Furthermore, the transcript expression levels of PI3, MMP8, CCR2, and PTX3 involved in the onset of KD vasculitis and IVIG resistance, as well as factors induced by TNF- $\alpha$ stimulation decreased significantly after IFX therapy.

$\mathrm{PI} 3$, also known as skin-derived antileukoproteinase, elafin, and trappin-2, is a potent serine protease inhibitor that prevents excessive damage under inflammatory and immune responses (22). This serine protease inhibitor, which is derived from epithelial cells and macrophages via TNF- $\alpha$ and IL- $1 \beta$ stimulation, exerts various biological effects including immunity modulation, e.g., neutrophil recruitment and nuclear factor-kappa B activation, and antiprotease activities, such as inhibitory effects on neutrophil elastase, the action of which induces tissue damage under inflammatory conditions.

Neutrophil collagenase (MMP8) of the MMP family is thought to be synthesized exclusively by neutrophils and plays a key role in the degradation of extracellular matrix constituents (17). Recently, the expression of the MMP8 gene has been observed among IVIG-resistant subjects, and the serum levels of MMP8 have been reported to be significantly higher in the acute phase of KD (13). In addition, the expression of MMP8 induced via TNF- $\alpha$ stimulation of mice cardiac cells has been demonstrated (23).

CCR2 is the receptor for the CC chemokine known as monocyte chemotactic protein 1 . Monocyte chemotactic protein 1 is produced by various cells following stimulation with TNF- $\alpha$ or IL- $1 \beta$. The monocyte chemotactic protein 1 levels have been reported to be elevated during the acute phase of $\mathrm{KD}(24)$. The role of CCR2 has also been reported in an animal model as 
being important for the pathogenesis of coronary vasculitis in KD (25). Furthermore, the administration of IFX therapy reduces the serum levels of monocyte chemotactic protein 1 and CCR2 in patients with rheumatoid arthritis (26).

Pentraxins constitute a super family of conserved proteins characterized by the pentraxin domain. PTX3 reflects the degree of inflammation of the local vasculature in response to the actions of TNF- $\alpha$ and IL- $1 \beta$, compared with C-reactive protein, which is produced in the liver following IL-6 stimulation. The PTX3 levels are significantly high in IVIG-resistant KD subjects compared with those observed in IVIG-responsive subjects (27). Moreover, PTX3 has been shown to be a good biological marker for predicting the clinical outcomes of subjects with Takayasu's arteritis (28).

These four transcripts related to KD inflammation, IVIG resistance and factors induced by TNF- $\alpha$ stimulation were also more suppressed by IFX therapy in the independent cohort of IFX-responsive subjects with IVIG resistance, indicating that the administration of IFX is beneficial in IVIG-resistant subjects.

We recognize several limitations in our study. The timing of blood sampling was not matched between the IFX-responsive and IVIG-responsive subjects. Because $\mathrm{KD}$ is a very dynamic and variable disease over the course of the acute phase, variation in transcript abundance profiles is expected to be associated with differences in the illness day with respect to the timing of blood sampling. In addition, we consider that the transcript patterns were influenced by the different cell numbers in each sample, using whole blood samples. Transcript abundance profiles that were differentially abundant following IFX therapy as the third-line treatment were found to be definitively affected by IVIG therapy. Therefore, it was confirmed that transcript abundance profiles were changed more by IFX therapy compared with that of IVIG.

In conclusion, the administration of IFX was found to be associated with both the signaling pathways of KD inflammation and several transcripts related to IVIG resistance factors. These findings provide strong theoretical support for the use of IFX in KD patients with IVIG resistance.

\section{METHODS}

\section{Subjects and Sample Collection}

Whole blood samples for the microarray study were obtained from eight IVIG-resistant KD subjects, given an IFX preparation (Remicade: Mitsubishi Tanabe Pharma, Osaka, Japan) as third-line treatment. The samples were collected before the IFX and $36 \mathrm{~h}$ after the end of IFX therapy (Table 1). All subjects had been admitted to Kitasato University Hospital between 2007 and 2012, were Japanese and fulfilled the criteria for the Diagnostic Guidelines for Kawasaki Disease (5th revision) published by the Kawasaki Disease Research Committee in Japan (29). The subjects were given a total dose of $4 \mathrm{~g} /$ $\mathrm{kg}$ of IVIG as the initial and second-line therapy before the IFX therapy. However, they exhibited resistance to the IVIG therapy and were therefore subsequently treated with a dose of $5 \mathrm{mg} / \mathrm{kg}$ of IFX infused over $2 \mathrm{~h}$ as the third-line treatment between illness days 8 and 12 (median, 10.5 days). We defined resistance to therapy as the presence of a persistent or recurrent fever lasting $\geq 36 \mathrm{~h}$ after the completion of the IVIG or IFX therapy. All eight subjects responded to IFX therapy following two cycles of IVIG therapy; their fever subsided within $36 \mathrm{~h}$ and other symptoms disappeared after administration of IFX. Oral aspirin $(30 \mathrm{mg} / \mathrm{kg} / \mathrm{d})$ was given to all subjects during the acute phase. Two-dimensional echocardiograms were performed on all subjects before initial IVIG and at $36 \mathrm{~h}$ after each treatment and at $1 \mathrm{mo}$ after the end of each treatment. The internal diameter of the coronary artery was measured at the main trunk coronary artery, the proximal left anterior descending, and the right coronary artery. Body surface area was computed by Haycock calculating formula (30). The coronary artery $Z$-score was computed by the Dallaire calculating formula (31). We defined $Z$-max as the maximum Z-score in any coronary artery segments during 1 moafter onset $\mathrm{KD}$. CAAs were defined as the maximum $Z$-score $\geq 5$.

To identify the transcript profiles that were differentially abundant after IFX therapy and exclude those that were differentially abundant after IVIG therapy, we obtained mRNA from the whole blood samples of six initial IVIG-responsive subjects before the IVIG therapy and $36 \mathrm{~h}$ after the end of IVIG therapy. These subjects were treated with a dose of $2 \mathrm{~g} / \mathrm{kg}$ IVIG infused over $24 \mathrm{~h}$ as the initial therapy between illness days 2 and 6 (median, 5.5 days) (Supplementary Table S2 online). Sex, age, and illness days at diagnosis were similar between these subjects and the IFX-responsive subjects. We first identified the transcripts that were differentially abundant between the pre- and post-IFX therapy periods and compared them with the transcripts of the subjects exhibiting an initial IVIG response. Among these transcripts, we then obtained those associated with the onset of KD vasculitis, IVIG resistance, and factors induced by TNF- $\alpha$ stimulation (Figure 1).

Subsequently, to validate the microarray results, we analyzed the expression levels of the quantitative RT-PCR transcripts using whole blood samples obtained from independent cohorts of nine KD subjects who exhibited an IFX response. These subjects were also given IVIG twice (total $4 \mathrm{~g} / \mathrm{kg}$ ) before IFX therapy and treated with IFX as the third-line treatment within 12 illness days (median, 11 days). All nine subjects responded well to the IFX therapy. Blood samples were also collected before the IFX and $36 \mathrm{~h}$ after the end of the IFX therapy.

Informed consent was obtained from the parents of each child enrolled in this study, and the study design was approved by the ethics review board at Kitasato University Hospital.

\section{RNA Isolation, cDNA Preparation, and Hybridization}

Whole blood was collected in PAX gene tubes, followed by total RNA isolation and CDNA preparation, and hybridization, as previously described (32). The results of the GeneChip analysis can be found in the NCBI's Gene Expression Omnibus and are accessible through the Gene Expression Omnibus Series accession database.

\section{Microarray Analysis}

For the expression analysis of the gene chip microarray, the CEL files created from each gene chip were imported into the GeneSpring GX 12.5 software program (Agilent Technologies, Santa Clara, CA, USA). Normalization and baseline approach was performed using the robust multi-array average and median of all samples (33). To exclude poorly reproducible transcripts, probes with an expression level lower than $20 \%$ were removed from the experiment based on the signal intensity value. Comparisons of the values obtained before and after each therapy were made using the Mann-Whitney $U$-test. Multiple comparisons of the transcript data for each group were corrected according to the Benjamini Hochberg false discovery rate ( $Q$ value). Values of $P<0.05$ and $Q<0.05$ were considered to be statistically significant. To identify the differential transcript abundance between the two groups of samples, a filter of $>1.5$-fold was applied. A pathway analysis was performed using WikiPathways (http://www.wikipathways.org) to search for biological pathways by importing the data into the GeneSpring GX 12.5 software program (Agilent Technologies). WikiPathways is a resource for biological pathways in the form of a Wiki. It serves as a repository for biological knowledge in the form of a pathway diagram and a platform for curating, sharing, and publishing pathways (34).

\section{Quantitative Real-Time PCR}

The transcript levels for PI3, MMP8, CCR2, and PTX3 were measured using a TaqMan Gene Expression assay (PI3; Hs0016066_m1, MMP8; Hs01029060_m1, CCR2; Hs00356601_m1, PTX3; Hs00173615_m1, 
Life Technologies, Carlsbad, CA). Each transcript expression level was normalized to that of the housekeeping gene, TATA box binding protein-associated factor RNA polymerase 1B (Hs01057259_m1, Life Technologies), and calculated using the comparative CT method.

\section{Statistical Analysis}

The analyses of the clinical variables were conducted using the SPSS statistical software package, version 15 (SPSS Japan, Tokyo, Japan). For the subject characteristics, laboratory data and quantitative RT-PCR data, the Mann-Whitney $U$-test, and Fisher's exact test were used to compare samples in each group. The Wilcoxon signed-rank test was used to compare the data obtained before and after each therapy. For the gene chip microarray data, the Mann-Whitney $U$-test was performed to compare the values obtained before and after each therapy, and the $P$ value in the pathway analysis was calculated according to Fisher's exact test using the GeneSpring GX 12.5 software package (Agilent Technologies). A value of $P<0.05$ was considered to be statistically significant.

\section{SUPPLEMENTARY MATERIAL}

Supplementary material is linked to the online version of the paper at http:// www.nature.com/pr

\section{ACKNOWLEDGMENTS}

We thank Robert E Brandt, Founder, CEO, and CME, of MedEd Japan, for editing the manuscript.

\section{STATEMENT OF FINANCIAL SUPPORT}

This work was supported by a Parents' Association Grant from the Kitasato University School of Medicine, a grant-in-aid from the Morinaga Foundation for Health and Nutrition and a grant-in-aid from the Kawasaki Disease Research Center in Japan.

Disclosure: The authors declare no conflict of interest.

\section{REFERENCES}

1. Newburger JW, Takahashi M, Gerber MA, et al.; Committee on Rheumatic Fever, Endocarditis, and Kawasaki Disease, Council on Cardiovascular Disease in the Young, American Heart Association. Diagnosis, treatment, and long-term management of Kawasaki disease: a statement for health professionals from the Committee on Rheumatic Fever, Endocarditis, and Kawasaki Disease, Council on Cardiovascular Disease in the Young, American Heart Association. Pediatrics 2004;114:1708-33.

2. Matsubara T, Furukawa S, Yabuta K. Serum levels of tumor necrosis factor, interleukin 2 receptor, and interferon-gamma in Kawasaki disease involved coronary-artery lesions. Clin Immunol Immunopathol 1990;56:29-36.

3. Furukawa S, Matsubara T, Umezawa Y, Okumura K, Yabuta K. Serum levels of p60 soluble tumor necrosis factor receptor during acute Kawasaki disease. J Pediatr 1994;124(5 Pt 1):721-5.

4. Hui-Yuen JS, Duong TT, Yeung RS. TNF-alpha is necessary for induction of coronary artery inflammation and aneurysm formation in an animal model of Kawasaki disease. J Immunol 2006;176:6294-301.

5. Siddiqui MA, Scott LJ. Spotlight on infliximab in Crohn disease and rheumatoid arthritis. BioDrugs 2006;20:67-70.

6. Burns JC, Mason WH, Hauger SB, et al. Infliximab treatment for refractory Kawasaki syndrome. J Pediatr 2005;146:662-7.

7. Burns JC, Best BM, Mejias A, et al. Infliximab treatment of intravenous immunoglobulin-resistant Kawasaki disease. J Pediatr 2008;153:833-8.

8. Son MB, Gauvreau K, Burns JC, et al. Infliximab for intravenous immunoglobulin resistance in Kawasaki disease: a retrospective study. J Pediatr 2011;158:644-649.e1.

9. Hirono K, Kemmotsu Y, Wittkowski H, et al. Infliximab reduces the cytokine-mediated inflammation but does not suppress cellular infiltration of the vessel wall in refractory Kawasaki disease. Pediatr Res 2009;65:696-701.

10. Maury CP, Salo E, Pelkonen P. Circulating interleukin-1 beta in patients with Kawasaki disease. N Engl J Med 1988;319:1670-1.

11. Suganami Y, Kawashima H, Hasegawa D, Sato S, Hoshika A. Clinical application of rapid assay of serum interleukin-6 in Kawasaki disease. Pediatr Int 2008;50:264-6.
12. Sohn MH, Noh SY, Chang W, Shin KM, Kim DS. Circulating interleukin 17 is increased in the acute stage of Kawasaki disease. Scand J Rheumatol 2003;32:364-6.

13. Fury W, Tremoulet AH, Watson VE, et al. Transcript abundance patterns in Kawasaki disease patients with intravenous immunoglobulin resistance. Hum Immunol 2010;71:865-73.

14. Ikeda K, Yamaguchi K, Tanaka T, et al. Unique activation status of peripheral blood mononuclear cells at acute phase of Kawasaki disease. Clin Exp Immunol 2010;160:246-55.

15. Popper SJ, Shimizu C, Shike H, et al. Gene-expression patterns reveal underlying biological processes in Kawasaki disease. Genome Biol 2007;8:R261.

16. Takahashi Y, Isuzugawa K, Murase Y, et al. Up-regulation of NOD1 and NOD2 through TLR4 and TNF-alpha in LPS-treated murine macrophages. J Vet Med Sci 2006;68:471-8.

17. Ra HJ, Parks WC. Control of matrix metalloproteinase catalytic activity. Matrix Biol 2007;26:587-96.

18. Ikeda $\mathrm{K}$, Ihara $\mathrm{K}$, Yamaguchi $\mathrm{K}$, et al. Genetic analysis of MMP gene polymorphisms in patients with Kawasaki disease. Pediatr Res 2008;63:182-5.

19. Park JA, Shin KS, Kim YW. Polymorphism of matrix metalloproteinase-3 promoter gene as a risk factor for coronary artery lesions in Kawasaki disease. J Korean Med Sci 2005;20:607-11.

20. Shimizu C, Jain S, Davila S, et al. Transforming growth factor-beta signaling pathway in patients with Kawasaki disease. Circ Cardiovasc Genet 2011;4:16-25.

21. Shimizu C, Oharaseki T, Takahashi K, Kottek A, Franco A, Burns JC. The role of TGF- $\beta$ and myofibroblasts in the arteritis of Kawasaki disease. Hum Pathol 2013;44:189-98.

22. Verrier T, Solhonne B, Sallenave JM, Garcia-Verdugo I. The WAP protein Trappin-2/Elafin: a handyman in the regulation of inflammatory and immune responses. Int J Biochem Cell Biol 2012;44:1377-80.

23. Awad AE, Kandalam V, Chakrabarti S, et al. Tumor necrosis factor induces matrix metalloproteinases in cardiomyocytes and cardiofibroblasts differentially via superoxide production in a PI3Kgamma-dependent manner. Am J Physiol Cell Physiol 2010;298:C679-92.

24. Terai M, Jibiki T, Harada A, et al. Dramatic decrease of circulating levels of monocyte chemoattractant protein-1 in Kawasaki disease after gamma globulin treatment. J Leukoc Biol 1999;65:566-72.

25. Martinez HG, Quinones MP, Jimenez F, et al. Important role of CCR2 in a murine model of coronary vasculitis. BMC Immunol 2012;13:56.

26. Xia L, Lu J, Xiao W. Blockage of TNF- $\alpha$ by infliximab reduces CCL2 and CCR2 levels in patients with rheumatoid arthritis. J Investig Med 2011;59:961-3.

27. Katsube $Y$, Akao M, Watanabe M, et al. Ptx3, a new biomarker for vasculitis, predicts intravenous immunoglobulin unresponsiveness in patients with kawasaki disease. J Am Coll Cardiol 2011;57:E2038.

28. Ishihara T, Haraguchi G, Kamiishi T, Tezuka D, Inagaki H, Isobe M. Sensitive assessment of activity of Takayasu's arteritis by pentraxin3, a new biomarker. J Am Coll Cardiol 2011;57:1712-3.

29. Ayusawa M, Sonobe T, Uemura S, et al.; Kawasaki Disease Research Committee. Revision of diagnostic guidelines for Kawasaki disease (the $5^{\text {th }}$ revised edition). Pediatr Int 2005;47:232-4.

30. Haycock GB, Schwartz GJ, Wisotsky DH. Geometric method for measuring body surface area: a height-weight formula validated in infants, children, and adults. J Pediatr 1978;93:62-6.

31. Dallaire F, Dahdah N. New equations and a critical appraisal of coronary artery Z scores in healthy children. J Am Soc Echocardiogr 2011;24:60-74.

32. Ogata S, Ogihara $Y$, Nomoto K, et al. Clinical score and transcript abundance patterns identify Kawasaki disease patients who may benefit from addition of methylprednisolone. Pediatr Res 2009;66:577-84.

33. Irizarry RA, Bolstad BM, Collin F, Cope LM, Hobbs B, Speed TP. Summaries of Affymetrix GeneChip probe level data. Nucleic Acids Res 2003;31:e15.

34. Kelder T, van Iersel MP, Hanspers K, et al. WikiPathways: building research communities on biological pathways. Nucleic Acids Res 2012;40(Database issue):D1301-7. 